\title{
Quantum anomalous Hall effect in two-dimensional magnetic insulator heterojunctions
}

\author{
Jinbo Pan ${ }^{1,6}$, Jiabin $\mathrm{Yu}^{2,3,6}$, Yan-Fang Zhang ${ }^{1,4,5}$, Shixuan $\mathrm{Du}^{4}$, Anderson Janotti $\mathbb{i D}^{5}$, Chao-Xing Liu (iD) ${ }^{2 凶}$ and Qimin Yan (iD ${ }^{1 凶}$
}

Recent years have witnessed tremendous success in the discovery of topological states of matter. Particularly, sophisticated theoretical methods in time-reversal-invariant topological phases have been developed, leading to the comprehensive search of crystal database and the prediction of thousands of topological materials. In contrast, the discovery of magnetic topological phases that break time reversal is still limited to several exemplary materials because the coexistence of magnetism and topological electronic band structure is rare in a single compound. To overcome this challenge, we propose an alternative approach to realize the quantum anomalous Hall (QAH) effect, a typical example of magnetic topological phase, via engineering two-dimensional (2D) magnetic van der Waals heterojunctions. Instead of a single magnetic topological material, we search for the combinations of two 2D (typically trivial) magnetic insulator compounds with specific band alignment so that they can together form a type-III brokengap heterojunction with topologically non-trivial band structure. By combining the data-driven materials search, first-principles calculations, and the symmetry-based analytical models, we identify eight type-III broken-gap heterojunctions consisting of 2D ferromagnetic insulators in the MXY compound family as a set of candidates for the QAH effect. In particular, we directly calculate the topological invariant (Chern number) and chiral edge states in the MnNF/MnNCl heterojunction with ferromagnetic stacking. This work illustrates how data-driven material science can be combined with symmetry-based physical principles to guide the search for heterojunction-based quantum materials hosting the QAH effect and other exotic quantum states in general.

npj Computational Materials (2020)6:152; https://doi.org/10.1038/s41524-020-00419-y

\section{INTRODUCTION}

The quantum anomalous Hall (QAH) effect ${ }^{1-3}$, a zero magnetic field manifestation of the integer quantum Hall effect, originates from the exchange interaction between electron spin and magnetism and exhibits quantized Hall resistance and zero longitudinal resistance. Similar to the quantum Hall effect, the QAH effect harbors dissipationless chiral edge states, thus providing an energyefficient platform for state-of-the-art applications in spintronics ${ }^{4,5}$ and quantum computing ${ }^{6,7}$. Guided by theoretical predictions ${ }^{8}$, the QAH effect was experimentally demonstrated in the magnetically $(\mathrm{Cr}$ or $\mathrm{V})$ doped $(\mathrm{Bi}, \mathrm{Sb})_{2} \mathrm{Te}_{3}{ }^{9-12}$. However, magnetic doping reduces the spin-orbit coupling (SOC) strength and thus may drive the system into a trivial phase. It also degrades the sample quality by bringing a large amount of disorder scatterings into the system. Consequently, the critical temperature of the QAH state through the magnetic doping approach is usually below $2 \mathrm{~K}$, an order-ofmagnitude lower than the Curie temperature of these compounds. Therefore, it is highly desirable to search for new platforms consisting of stoichiometric materials with intrinsic magnetism to realize high temperature $\mathrm{QAH}$ state.

Recent rapid development of two-dimensional (2D) layered magnetic insulators has provided an exciting opportunity for the realization of QAH effect in un-doped material systems. The $\mathrm{QAH}$ effect has been proposed to appear in the heterostructures of topological insulator (TI) and magnetic insulator through the magnetic proximity effect across the interface ${ }^{13-17}$. Another recent advance is the successful synthesis of a class of intrinsic magnetic TI materials, including $\mathrm{MnBi}_{2} \mathrm{Te}_{4}, \mathrm{MnBi}_{2} \mathrm{Se}_{4}$, and $\mathrm{MnSb}_{2} \mathrm{Te}_{4}{ }^{18,19}$, in which A-type antiferromagnetism and topologically nontrivial band structure coexist, leading to the successful observation of the QAH state ${ }^{20-22}$. The QAH effect has also been observed in twisted bilayer graphene ${ }^{23,24}$.

\section{RESULTS}

Discovery workflow for QAH heterojunctions

Despite several recent works ${ }^{25,26}$, the search for more QAH materials is still challenging because it requires the coexistence of ferromagnetism and topological band structure, which is rare in a single material. In this study, we propose an alternative strategy to realize the $\mathrm{QAH}$ effect in $2 \mathrm{D}$ magnetic van der Waals (VdW) heterojunctions by combining data-driven discovery of $2 \mathrm{D}$ magnetic compounds with a theoretical analysis of topological properties. All the previous works ${ }^{13-16,25,26}$ on the QAH heterojunctions require at least one intrinsic topological material. In contrast, every QAH VdW heterojunction predicted by our approach can be constructed with two trivial 2D ferromagnetic (FM) insulators. There are three types of heterojunctions, defined by the band alignment between the two constituting materials, as shown in Supplementary Fig. 1. The key idea is to make sure the two FM 2D materials form the so-called type-III broken-gap junction, in which the conduction-band minimum (CBM) of one $2 \mathrm{D}$ material is lower in energy than the valence-band maximum (VBM) of the other compound at certain high-symmetry momentum, as shown in Fig. 1a. In other words, an inverted (topological) band structure is formed by the heterojunction, rather than one individual material. As this strategy only requires the (valence or conduction) band offset to be larger than the band gap of one of

\footnotetext{
${ }^{1}$ Department of Physics, Temple University, Philadelphia, PA 19122, USA. ${ }^{2}$ Department of Physics, The Pennsylvania State University, University Park, PA 16802, USA. ${ }^{3}$ Condensed Matter Theory Center, Department of Physics, University of Maryland, College Park, Maryland 20742, USA. ${ }^{4}$ Institute of Physics, Chinese Academy of Science, Beijing 100190, China. ${ }^{5}$ Department of Materials Science and Engineering, University of Delaware, DE 19716 Wilmington, USA. ${ }^{6}$ These authors contributed equally: Jinbo Pan, Jiabin Yu.

凶email: cxl56@psu.edu; qiminyan@temple.edu
} 
(a)

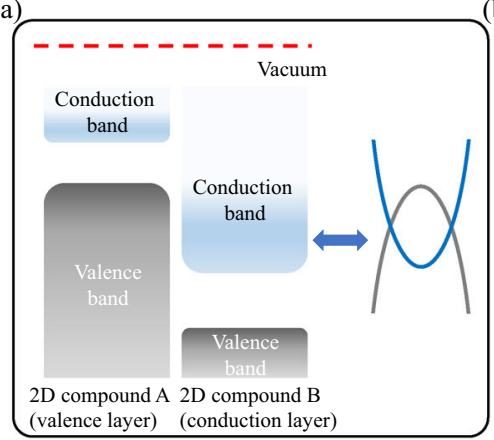

(b)

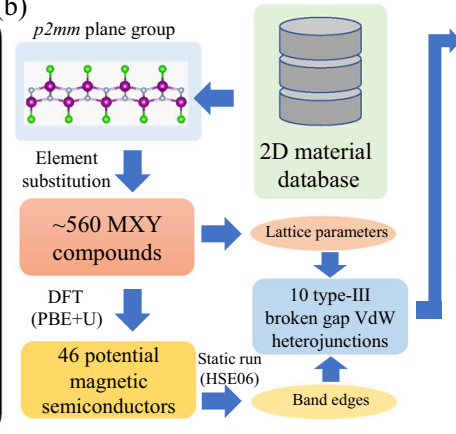

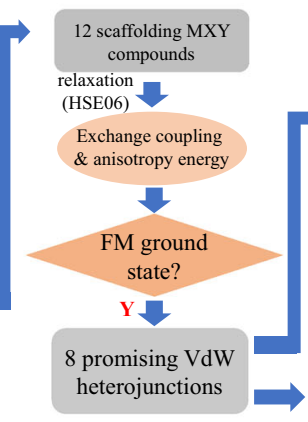

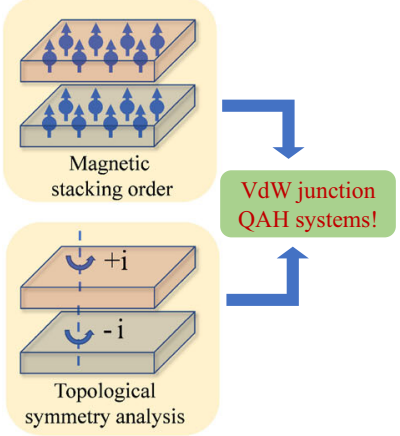

Fig. 1 Illustration of a data-driven materials discovery and design framework that combines high-throughput computations and symmetry-based analysis for the search for heterojunction-based 2D magnetic topological systems that host the QAH effect. a Schematic of hypothesis: QAH effect that can be emerged from type-III broken-gap 2D VdW heterojunctions. b Data-driven discovery pipeline for VdW heterojunction-based QAH systems.

the two 2D materials, it greatly relaxes the stringent conditions for the QAH effect and allows us to develop a systematic approach to identify the candidate compounds for the QAH heterojunctions in 2D magnetic VdW material database.

This approach has been successfully utilized to search for the quantum spin Hall insulators, such as InAs/GaSb quantum wells ${ }^{27}$ and other semiconductor heterojunctions ${ }^{28}$, but it has not been applied to the search of QAH systems, mainly due to the lack of 2D magnetic materials database. Therefore, a data-driven computational and theoretical search of 2D magnetic materials is called for. Figure $1 \mathrm{~b}$ summarizes the workflow for our data-driven computational approach, which is carefully designed to incorporate highthroughput calculations at multiple computational levels and symmetry-based physical principles that are critical to quickly identify the most promising candidates hosting the QAH effect. In this initial work, we focus on a family of $2 D$ monolayer $M X Y$ compounds ( $\mathrm{M}=$ metal atoms, $\mathrm{X}=\mathrm{N}, \mathrm{S}, \mathrm{Se}, \mathrm{Te}, \mathrm{Y}=\mathrm{F}, \mathrm{Cl}, \mathrm{Br}$, I) and predict 46 potential $2 \mathrm{D}$ magnetic semiconductors in this family. $A$ database including the information of CBM, VBM, band offset, the orbital nature and the symmetry properties of the conduction and valence bands, and the magnetic anisotropy energies and exchange coupling for each candidate compound is created. Based on this 2D magnetic material database, we identify eight broken-gap VdW heterojunctions made from different combination of these compounds to possess inverted band structure.

We next perform a generic symmetry analysis of topological properties for the VdW heterojunctions. This analysis is based on the relationship between topological invariant, the Chern number (CN) that characterizes the QAH effect, and the two-fold rotation $\left(C_{2}\right)$ eigenvalues ${ }^{29-33}$. We demonstrate that the $\mathrm{CN}$ for the heterojunction must be changed by an odd number when the magnetizations in two magnetic VdW compounds are switched from FM to anti-FM (AFM) stacking order. This suggests that the QAH phase must exist for at least one magnetic stacking order.

Finally, we choose MnNF/MnNCl heterojunctions as an example and construct a tight-binding model that captures the main features of the band structure from the realistic material simulations. Based on this tight-binding model, we explicitly compute the $\mathrm{CN}$ and the chiral edge states of this heterojunction. Our results show that the QAH phase occurs for the FM stacking order of the magnetization between two materials of the heterojunction and is consistent with our symmetry analysis by computing the $C_{2}$ eigenvalues from first-principles calculations.

\section{Prediction of 2D magnetic insulators in MXY compounds}

In the past decade, a tremendous amount of computed and experimental material data has been shared in the materials research community through multiple material databases for both bulk $^{34-36}$ and 2D inorganic compounds ${ }^{37-41}$. This offered a vast inorganic materials space that is explorable by data-driven approaches and enabled the data-driven discovery of quantum materials hosting exotic topological phases $25,26,42-44$. Here, our theoretical predictions of 2D heterojunctions that host the $\mathrm{QAH}$ effect is motivated by the data-driven discovery of a family of $2 \mathrm{D}$ monolayer $M X Y$ compounds $(M=$ metal atoms, $X=N, S, S e, T e$, $\mathrm{Y}=\mathrm{F}, \mathrm{Cl}, \mathrm{Br}, \mathrm{I})$ that are predicted to host both $\mathrm{FM}$ and nonmetallic ground states. We initiated our material discovery efforts from a material set of around 560 monolayer MXY compounds that are created through elemental substitution based on the existing MXY materials in public 2D materials databases ${ }^{37-41}$. The data-driven materials discovery process is illustrated in Fig. $1 \mathrm{~b}$ and more computational details can be found in Supplementary Fig. 2. With FM state as the initial magnetic configuration, through an energy minimization and electron density optimization process, we identified 46 potential magnetic semiconductors (see Supplementary Table 1). 2D FM insulators $\mathrm{MnNX}$ and $\mathrm{CrCX}(\mathrm{X}=\mathrm{Cl}, \mathrm{Br}, \mathrm{l} ; \mathrm{C}=\mathrm{S}$, $\mathrm{Se}, \mathrm{Te})$ have been predicted in previous work ${ }^{45-48}$. Our comprehensive study puts this $2 \mathrm{D}$ compound family as a great platform for the discovery and design of quantum materials based on $2 \mathrm{D}$ magnetic heterojunctions.

Constructed by ten different FM MXY compounds, we propose eight VdW heterojunctions (as listed in Table 1), in which the CBM of one 2D layer (called conduction layer) is below the VBM of the other 2D layer (called valence layer). Thus, all these heterojunctions exhibit the desirable band inversion, as well as small-enough $(<6.5 \%)$ lattice mismatch, as shown in Table 1 . The Curie temperatures $\left(T_{\mathrm{c}}\right)$ of the these 10 monolayer FM MXY compounds are evaluated by performing Monte-Carlo (MC) simulations based on a 2D Heisenberg Hamiltonian model ${ }^{49,50}$. The interaction types, the values of exchange coupling $J$ and magnetic anisotropy energy $A$ are summarized in Fig. 1a and Table 2. Computational details related to magnetic anisotropy energies and exchange coupling interactions are included in the Supplementary Methods 1 and Supplementary Fig. 3. Characterized by high Curie temperature as well as small band gaps, this set of magnetic semiconductor compounds provides a great platform for the design of VdW heterojunctions to realize the QAH effect. The CBM of all the conduction layers and the VBM of all the valence layers are both located at $\Gamma$ point, facilitating the analysis of broken-gap junctions.

Symmetry analysis for the VdW heterojunctions

We next analyze the topological properties of the proposed eight VdW heterojunctions based on the symmetry aspect. The magnetic moments are in the $z$ direction (normal to the 2D planes) for all these magnetic MXY compounds, which reduce 


\begin{tabular}{|c|c|c|c|c|c|c|c|c|}
\hline Material A & CBM $(\mathrm{eV})$ & $\begin{array}{l}\text { Band } \\
\text { character (CBM) }\end{array}$ & Material B & VBM (eV) & $\begin{array}{l}\text { Band } \\
\text { character (VBM) }\end{array}$ & $\begin{array}{l}\text { Lattice mismatch } \\
\text { along } a(\%)\end{array}$ & $\begin{array}{l}\text { Lattice mismatch } \\
\text { along } b(\%)\end{array}$ & $\begin{array}{l}\text { Nontrivial } \\
\text { stacking order }\end{array}$ \\
\hline FeNF & -7.9 & Fe: $d_{x}^{2}-y^{2}$ & $\mathrm{MnNF}$ & -7.6 & $\mathrm{~N}: p_{y}$ & 2.3 & 1.1 & FM \\
\hline VSI & -5.9 & $\mathrm{~V}: d_{x y}$ & MoSI & -5.6 & Mo: $d_{y z}$ & 1.7 & 3.2 & FM \\
\hline VSI & -5.9 & $\mathrm{~V}: d_{x y}$ & WSel & -4.8 & $\mathrm{~W}: d_{y z}$ & 3.9 & 6.1 & FM \\
\hline VSI & -5.9 & $\mathrm{~V}: d_{x y}$ & VSel & -5.6 & Se: $p_{x} /$ I: $p_{x}$ & 2.7 & 6.0 & FM \\
\hline MnNF & -7.1 & $\mathrm{Mn}: d_{x}^{2}-y^{2}$ & $\mathrm{MnNCl}$ & -6.7 & $\mathrm{~N}: p_{y}$ & 6.5 & 0.6 & $\mathrm{FM}$ \\
\hline TiTel & -4.9 & $\mathrm{Ti}: d z^{2}$ & WTel & -4.9 & $\mathrm{~W}: d_{y z}$ & 4.0 & 3.0 & FM \\
\hline vSel & -5.1 & $\mathrm{~V}: d z^{2} / d_{x}^{2-y}{ }^{2}$ & WSel & -4.8 & $\mathrm{~W}: d_{y z}$ & 6.5 & 0.1 & FM \\
\hline $\mathrm{CrSeCl}$ & -5.6 & Cr: $d_{x}^{2}-y^{2}$ & VSel & -5.6 & Se: $p_{x} /$ I: $p_{x}$ & 4.1 & 0.5 & FM \\
\hline
\end{tabular}

"Nontrivial stacking order" indicates the stacking order that guarantees a nonzero CN under the assumption that each individual material is topologically trivial. Valence-band maximum (VBM) and conduction-band minimum (CBM) are given with respect to vacuum level.

Table 2. Magnetic interaction parameters and Curie temperatures of MXY compounds.

\begin{tabular}{|c|c|c|c|c|c|c|c|}
\hline & $J_{12}$ & $J_{13}$ & $J_{34}$ & $A_{[100]}$ & $A_{[010]}$ & $A_{[001]}$ & Tc (K) \\
\hline TiTel & 9.33 & -13.19 & -1.22 & $0.001 *$ & $0.001 *$ & 0 & 30 \\
\hline FeNF & 0.09 & -19.85 & -8.90 & $-0.02 *$ & $0.38 *$ & 0 & 459 \\
\hline VSI & -93.41 & -73.03 & -43.59 & 1.85 & 1.27 & 0 & 1100 \\
\hline VSel & -7.03 & -34.49 & -24.83 & 2.89 & 1.57 & 0 & 913 \\
\hline $\mathrm{MnNF}$ & -16.39 & -8.73 & -2.46 & 0.06 & 0.07 & 0 & 671 \\
\hline $\mathrm{MnNCl}$ & -16.13 & -5.67 & -2.91 & 0.06 & 0.11 & 0 & 592 \\
\hline $\mathrm{CrSeCl}$ & -3.79 & -6.13 & 1.69 & -0.06 & 0.002 & 0 & 270 \\
\hline MoSI & 1.07 & -3.08 & 1.07 & -0.08 & -0.45 & 0 & 66 \\
\hline WSel & 4.03 & -7.68 & -6.38 & 1.32 & -0.41 & 0 & 318 \\
\hline WTel & 2.12 & -11.86 & -6.26 & -0.10 & -0.91 & 0 & 517 \\
\hline
\end{tabular}

Exchange coupling parameter $J$ (in meV), magnetic anisotropy energy $A$ (in $\mathrm{meV}$ ), and estimated Curie temperature Tc of MXY compounds based on hybrid function (HSE06) calculations.

*Magnetic anisotropy energies for TiTel and FeNF were evaluated using the $\mathrm{PBE}+\mathrm{U}$ method, as the HSEO6 calculations did not converge well for these two compounds.

their plane groups to $p 2$, generated by $C_{2}$ and lattice translations. Putting two 2D materials together to form a heterojunction can preserve the $p 2$ symmetry as long as the magnetic moments of two layers are in the same or opposite directions (named as FM or AFM stacking, respectively). Using $C_{2}$ eigenvalues ${ }^{29-33}$, we will argue that the two stacking orders result in different $\mathrm{CNs}$ once the heterojunctions are in the inverted regime, meaning that at least one way gives $\mathrm{QAH}$ heterojunctions.

Owing to $C_{2}^{2}=-1$, the states at four $C_{2}$-invariant momenta have definite $C_{2}$ eigenvalues $\pm i$, as exemplified in Fig. 2 . The quantity of interest is the total number $n_{-}$of occupied states with $C_{2}$ eigenvalue $-i$ at all $C_{2}$-invariant momenta, as

$\mathrm{CN} \bmod 2=n_{-} \bmod 2$

holds for a 2D insulator ${ }^{29,30}$. To get a clear physical picture, we first neglect the SOC and the interlayer coupling between two layers in the VdW heterojunctions but include the charge transfer that equalizes the chemical potentials of the two layers. Such approximation allows us to treat spin and layer indexes as good quantum numbers (see Fig. $2 \mathrm{a}, \mathrm{b}$ ), and then we can split $n_{-}$into $n_{-}=n_{-, v}+n_{-, c}$ with $n_{-, c}$ and $n_{-, v}$ from the conduction and valence layers, respectively. The only difference between the FM and AFM stacking is the relative direction of magnetic moments in the two constituting compounds. Without loss of generality, we reverse the direction of the magnetic moment of the conduction layer when switching the stacking order. Since the magnetization in the valence layer stays fixed, we have $n_{-, v}^{F M}=n_{-. v}^{A F M}$ with the superscripts indicating the stacking order.

To address $n_{-, c}$, we further split $n_{-, c}$ into $n_{-, c}=n_{-, \text {, low }}+$ $n_{-, c, h i g h}$, where $n_{-, c, \text { low }}$ comes from the band that is partially filled (involved in the band inversion) and $n_{-, c, \text { high }}$ is given by the occupied bands fully below $E_{F}$ (see Fig. $2 a$, b). In the conduction layer, the sign of $C_{2}$ eigenvalue of any state is flipped when switching from the FM stacking to the AFM stacking, as the orbital nature is unchanged while the spin direction is reversed. It results in $n_{-, c \text {,high }}^{\mathrm{AFM}}=4 N_{c, \text { high }}-n_{-, c \text {,high }}^{\mathrm{FM}}$ where the pre-factor 4 comes from the four $C_{2}$-invariant momenta and $N_{c \text {,high }}$ is the number of occupied bands fully below $E_{\mathrm{F}}$ in the conduction layer. Since only one band from the conduction layer is partially filled and it only contributes to $n_{-, c}$ at $\Gamma$ for the heterojunctions of interest, $n_{-, \text {, low }}$ is either 0 or 1 , leading to $n_{-, c, \text { low }}^{\text {AFM }}=1-n_{-, c, \text { low }^{\mathrm{FM}} \text {. In sum, we have }}$ $n_{-}^{\mathrm{AFM}}=n_{-}^{\mathrm{FM}}+1+4 N_{c, \text { high }}-2 n_{-, c^{\prime}}^{\mathrm{FM}}$, resulting in

$n_{-}^{\mathrm{AFM}} \bmod 2=n_{-}^{\mathrm{FM}}+1 \bmod 2$.

Now we discuss the SOC and the interlayer coupling. The energy scales of these two effects are typically much smaller than the energy scale of the band inversion (i.e., the energy gap at $\Gamma$ in this case), which is determined by the hopping and the magnetic exchange coupling. Indeed, as shown by the results from firstprinciple calculations (see details in the Supplementary Fig. 8), the SOC and interlayer coupling do not cancel the existing band inversion or introduce new band inversion in the heterostructures of interest, leaving Eq. (2) unchanged. However, the two effects are still important for our analysis since they open the gap at generic momenta (not $C_{2}$-invariant momenta) and enable us to combine Eq. (2) with Eq. (1). (See schematic examples in Fig. 2c, d). As a result, we conclude that the parity of the $\mathrm{CN}$ is changed when switching the stacking order of the 2D heterojunction (from FM to AFM or the inverse), and thus at least one of the stacking orders can give non-zero $\mathrm{CN}$.

The remaining question is which stacking order guarantee a nonzero $\mathrm{CN}$ for the heterojunctions of interest. Let us assume that the CNs of the two individual materials have the same parity, e.g., both materials have zero $\mathrm{CNs}$, so that the total $n_{-}$of the two materials must be even before forming the heterojunction. Then, the key lies on the $C_{2}$ eigenvalues of the two states involved in the band inversion at $\Gamma$, i.e., CBM of the conduction layer and VBM of the valence layer in this case. Specifically, if the two states have opposite $C_{2}$ eigenvalues for a certain stacking order, $n_{-}$of the 
(a)

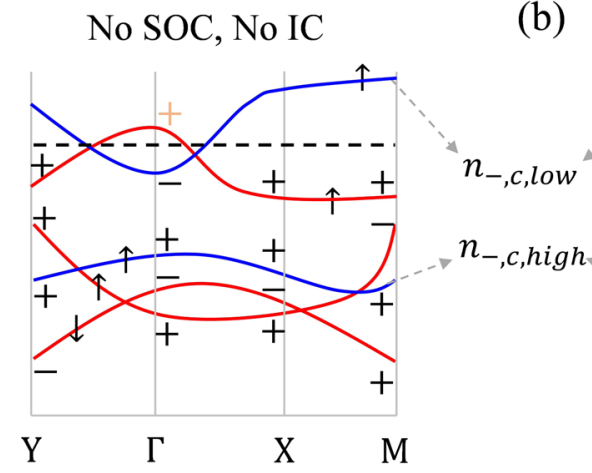

(b)

No SOC, No IC
FM Stacking : $n_{-}^{F M}=5$

(c)

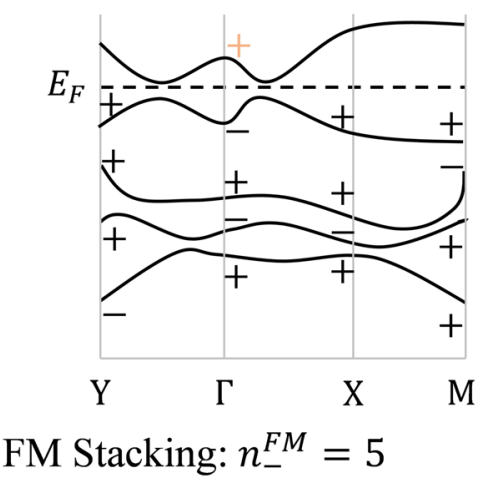

AFM Stacking : $n_{-}^{A F M}=8$

(d)

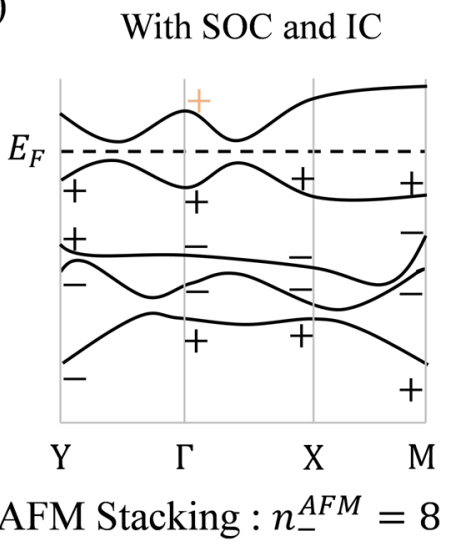

Fig. 2 Schematics of the examples with certain $n_{-}$that is correlated with the topological invariant. "IC" labels the interlayer coupling and the black dashed line is the Fermi energy. \pm correspond to the $C_{2}$ eigenvalues $\pm \mathrm{i}$, with the black labeling the occupied states and the orange labeling the empty states involved in the band inversion at $\Gamma$. In $\mathbf{a}, \mathbf{b}$, there are no SOC and no interlayer coupling, the blue and red lines come from the valence and conduction layers, respectively, and $\uparrow \downarrow$ stand for the spin polarization, whereas SOC and the interlayer coupling are included in $\mathbf{c}$ and $\mathbf{d}$. $\mathbf{a}$ and $\mathbf{c}$ are for FM stacking with $n_{-}^{F M}=5$, where $C N$ must be non-zero in $\mathbf{c}$ and the band inversion happens between two states with opposite $C_{2}$ eigenvalues. Flipping the spins in the conduction layer gives AFM stacking with $n_{-}^{A F M}=8$ as shown in $\mathbf{b}$ and $\mathbf{d}$. In this case, $\mathrm{CN}$ might be zero and the band inversion happens between two states with the same $C_{2}$ eigenvalues.

heterojunction must differ by 1 from the total $n_{-}$of the two materials before forming the heterojunction (see Fig. 2a). Thus, such stacking order must yield a QAH heterojunction according to Eq. (1). With this principle in mind, we list the $C_{2}$ eigenvalues of the VBM and CBM for all FM materials with all magnetic moments fixed in the positive $z$ direction (see Supplementary Table 2). From this list, we can directly read out the $C_{2}$ eigenvalues of the two states involved in the band inversion at the $\Gamma$ point for any heterojunction with FM stacking, and if they are opposite (same), the heterojunction has non-zero CN for the FM (AFM) stacking.

\section{The QAH effect in MnNF/MnNCl heterojunction}

The discussion above based on symmetry eigenvalues is valid for all the eight VdW heterojunctions identified if the magnetic moments are aligned along the $z$ direction, while the topological properties of heterojunctions with in-plane magnetization is discussed in the Supplementary Discussion 1 and Supplementary Fig. 4. From Table 2, we notice that the magnetic anisotropy of 5 compounds is out-of-plane while that of the other five materials is in-plane. For the heterojunction consisting of the compounds with in-plane easy axis, a small out-of-plane magnetic field may be required to align the magnetic moments. To conclusively demonstrate the emergence of $\mathrm{QAH}$ effect from these 2D magnetic VdW heterojunctions, we focus on a prototype system consisting of two magnetic compounds, $\mathrm{MnNF}$ and $\mathrm{MnNCl}$ with small band gaps. Both compounds are predicted to host FM ground state, being dynamically and thermally stable (Supplementary Figs. 5-7). The Curie temperatures of these two compounds are evaluated by both HSEO6 and PBE $+U$ functionals. We observe that the hybrid function predicts lower transition temperatures than $\mathrm{PBE}+\mathrm{U}$ for $\mathrm{MnNF}$ and $\mathrm{MnNCl}$, owing to the fact that the inclusion of exact exchange in HSEO6 functional generates more localized states on $\mathrm{Mn}$ atoms and weaker exchange interactions among them. Even in this case, the predicted Curie temperatures for $\mathrm{MnNF}$ and $\mathrm{MnNCl}$ are $671 \mathrm{~K}$ and $592 \mathrm{~K}$, respectively (Fig. 3a), well above room temperature. Both partial density of states and orbital-projected band structures of $\mathrm{MnNF}$ and $\mathrm{MnNCl}$ (Fig. 3b) indicate that the conduction-band edges of these compounds are mainly d states, while the valenceband edge is derived from $\mathrm{N} p$ y states.

Based on the calculated band alignment between the two compounds (as shown in Fig. 3b, c), we expect that a type-III broken-gap heterojunction can be formed with band inversion. As shown in Fig. 3d, we create $\mathrm{MnNF} / \mathrm{MnNCl} \mathrm{VdW}$ heterojunctions with both FM and AFM stacking orders. Atomic structures are relaxed using the hybrid functional (HSE06). The interlayer VdW interaction is evaluated by using the DFT-D3 correction method of Grimme et al. ${ }^{51}$. In the case of FM stacking, without SOC, the crossing between two bands in the same spin channel happens around $\Gamma$ point, leading to the band inversion of $14.0 \mathrm{meV}$ (Supplementary Fig. 8b), and this crossing is protected by mirror 
(a)

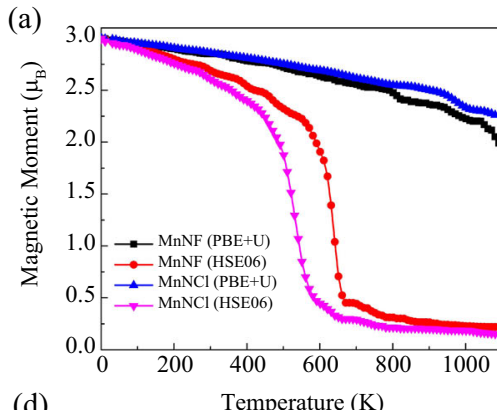

(d)

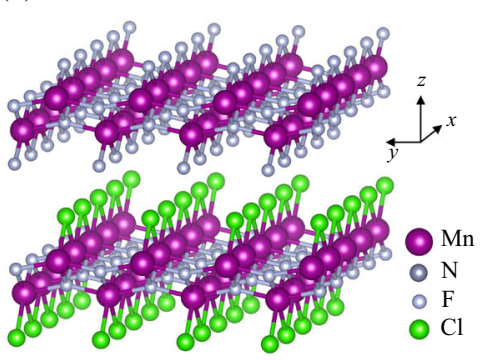

(b)

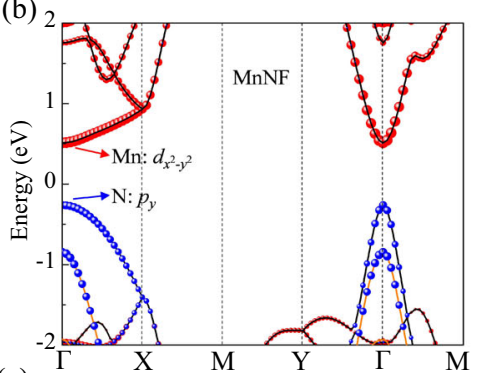

(e)

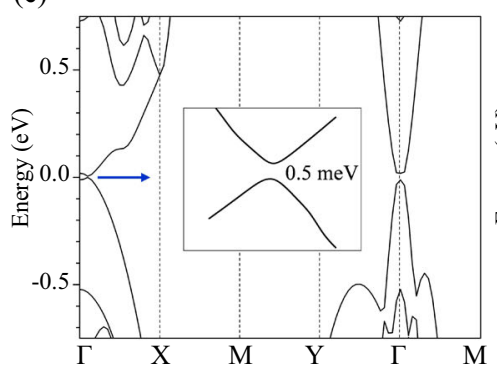

(c) 2

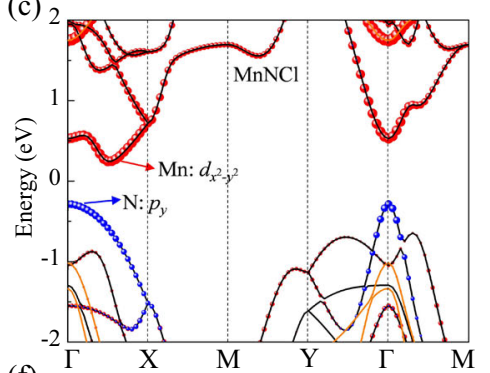

(f)

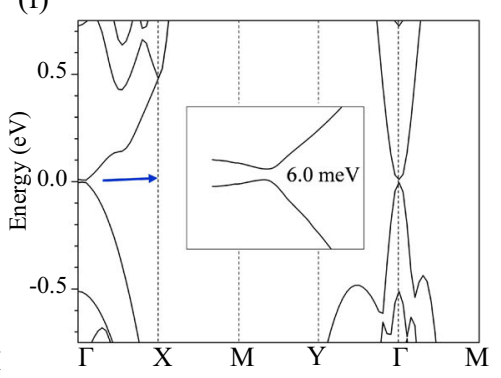

Fig. 3 Computational data for a representative material system: $\mathrm{MnNF}, \mathrm{MnNCl}$, and their VdW heterojunctions. a Estimation of Curie temperatures for $\mathrm{MnNF}$ and $\mathrm{MnNCl}$ by Monte-Carlo simulations based on a Heisenberg Hamiltonian model that is fitted to both HSEO6 and $\mathrm{PBE}+\mathrm{U}$ results. b, c Orbital-projected band structures of $\mathrm{MnNF}$ and $\mathrm{MnNCl}$; d The relaxed structure of $\mathrm{MnNF} / \mathrm{MnNCl}$ heterojunction. Band structures of $\mathrm{MnNF} / \mathrm{MnNCl}$ heterojunction (with SOC) with FM stacking order e without and $\mathbf{f}$ with a shift of the MnNF layer relative to the $\mathrm{MnNCl}$ layer by $0.5 \AA$ along the $y$ direction are shown.

symmetry. The inclusion of SOC can open band gaps at the original crossing points of the two bands located at the $\Gamma-X$ line. However, as the SOC is quenched by the crystal environment, this gap is only around $0.5 \mathrm{meV}$ (Fig. 3e). To enhance the gap, a relative shift of the MnNF layer relative to the $\mathrm{MnNCl}$ layer by $0.5 \AA$ along the $y$ direction is introduced to break the mirror symmetry (see detailed discussion in the Supplementary Discussion 2) and create a gap around $6.0 \mathrm{meV}$ (Fig. 3f). Furthermore, we observe that the band inversion at $\Gamma$ point can be controlled by applying a biaxial in-plane strain (through deformation potential effects) or an external electric field along $z$ direction (see Supplementary Fig. 9), thus providing effective ways to manipulate both electronic structures and topological properties of VdW heterojunctions. As compared to the strong intralayer magnetic interaction, the interlayer magnetic interaction is very weak. The total energy of the $\mathrm{MnNF} / \mathrm{MnNCl}$ heterojunction with FM stacking order is only $0.1 \mathrm{meV}$ per primitive cell lower than that with AFM stacking order. This combination of strong intralayer and weak interlayer magnetic couplings indicate that, once a FM (or AFM) stacking of the heterojunction is initialized, the magnetic stacking order will be rather stable, because the flip of one spin would be restrained by the strong intralayer magnetic interaction.

In $\mathrm{MnNF}$ and $\mathrm{MnNCl}$, within the $p 2 \mathrm{~mm}$ plane group, $\mathrm{Mn}$ cations are confined in a quasi-octahedral bonding environment associated with a $t_{2 \mathrm{~g}}-e_{\mathrm{g}} d$ state splitting. This crystal field splitting $(1 \sim 2 \mathrm{eV})$ provides an essential electronic structure scaffold for small-gap FM semiconductors. Orbital analysis confirms that the $\mathrm{CBM}$ of $\mathrm{MnNF}$ or $\mathrm{MnNCl}$ is composed of $\mathrm{Mn} e_{\mathrm{g}}$ states, whereas the VBM is derived from the interaction between occupied $\mathrm{N} p$ states and $M n t_{2 g}$ states. This unique electronic structure feature endows the VBM and CBM of MXY compounds different $C_{2}$ rotational eigenvalues (see Supplementary Tables 1 and 2), which is essential for the emergence of $\mathrm{QAH}$ effect in their heterojunctions. From our first-principles calculations, the CBM and VBM at the $\Gamma$ point (zone center) of $\mathrm{MnNF}$ and $\mathrm{MnNCl}$ have opposite $C_{2}$ eigenvalues in the FM configuration, indicating that the heterojunction with FM stacking must have non-zero $\mathrm{CN}$ according to the above symmetry analysis. To better understand the result of first-principle calculation, we construct effective models around $\Gamma$ point near the Fermi energy. Near $E_{\mathrm{F}}$, the conduction and valence bands of both materials are composed of $p_{y}$ and $d_{x 2-y_{2}}$ orbitals, respectively (as shown in Fig. $3 b, c ; x, y, z$ directions are defined in Fig. 3d). For the FM stacking, the basis can be chosen as $\left(\left|d_{x^{2}-y^{2}}, \uparrow\right\rangle,\left|p_{y}, \uparrow\right\rangle\right)$, resulting in the effective model:

$$
\left(\begin{array}{cc}
E_{v}+B_{v x} k_{x}^{2}+B_{v y} k_{y}^{2} & -i A_{y} k_{y}+\lambda_{1} k_{x} \\
i A_{y} k_{y}+\lambda_{1} k_{x} & E_{c}+B_{c x} k_{x}^{2}+B_{c y} k_{y}^{2}
\end{array}\right) .
$$

Here $\lambda_{1}$ is given by SOC, all other parameters are dominated by the hopping. More details are provided in the Supplementary Methods 2. According to the band structure from first-principles calculations, we may choose $E_{v}-E_{c}>0, B_{v x}-B_{c x}<0$, and $B_{v y}-B_{c y}<0$. Then, the direct calculation of the $C N$ yields $C N_{F M}^{\text {eff }}=-\operatorname{sgn}\left(A_{y} \lambda_{1}\right)$, which is non-zero. On the other hand, the effective model for AFM stacking has the form:

$$
\left(\begin{array}{cc}
E_{v}+B_{v x} k_{x}^{2}+B_{v y} k_{y}^{2} & -i \lambda_{0} \\
i \lambda_{0} & E_{c}+B_{c x} k_{x}^{2}+B_{c y} k_{y}^{2}
\end{array}\right) .
$$

with bases $\left(\left|d_{x^{2}-y^{2}}, \uparrow\right\rangle,\left|p_{y}, \downarrow\right\rangle\right)$ and $\lambda_{0}$ induced by SOC. The $k$ independent off-diagonal term leads to $C N$ equals zero. As the occupied bands fully below $E_{\mathrm{F}}$ have the same $C N$ s for different stacking orders, the total CNs should differ by one, confirming the above conclusion.

We then construct a tight-binding model for further clarification of the topological properties of the heterostructure. The basis of the model is given by the $d_{x-y}{ }^{2}, d_{x y}$ and $d_{y z}$ orbitals of the Mn atoms and the $p_{y}$ orbital of the $\mathrm{N}$ atoms. The FM of each layer is included by adding the localized magnetic moments on the $\mathrm{Mn}$ atoms and the on-site SOC is also included. With reasonable parameter values, the bulk band structures of the FM and AFM stacked heterostructures are shown in Fig. 4a, b, respectively, which qualitatively match those obtained by the first-principle calculations (see the Supplementary Methods 2 and Supplementary Fig. 10 for more details). Direct calculations for the FM and AFM stacked heterostructure yield $\mathrm{CN}=1$ and $\mathrm{CN}=0$, respectively, coinciding with the chiral edge mode for FM stacking in 

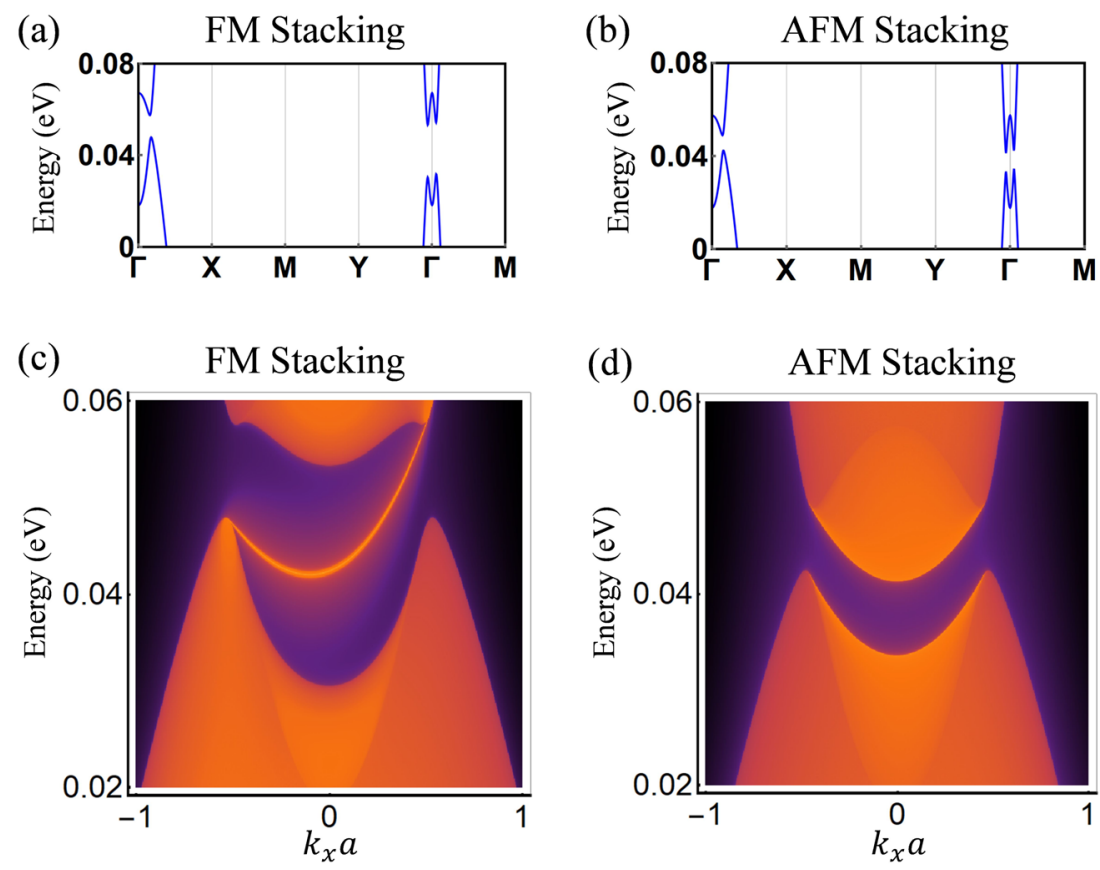

Fig. 4 Electronic band structures of $\mathrm{MnNF} / \mathrm{MnNCl}$ broken-gap VdW junctions generated by the tight-binding model. $a$, $b$ The bulk band structure and $\mathbf{c}$, d illustrate the energy dispersion along the (01) edge. In $\mathbf{c}, \mathbf{d}, a$ is the lattice constant along $x$ direction.

Fig. 4c and the fully gapped edge for AFM stacking in Fig. 4d. The tight-binding model calculation verifies different topological properties of the FM and AFM stacked heterostructures.

\section{DISCUSSION}

In summary, we proposed a new approach to realize the QAH effect in $2 \mathrm{D}$ magnetic $\mathrm{VdW}$ heterojunctions and conclusively demonstrate the existence of the QAH effect in $\mathrm{MnNF} / \mathrm{MnNCl}$ heterojunction with FM stacking by combining the first-principles calculations and the symmetry analysis. We also proposed other VdW heterojunctions that can host the QAH effect, as listed in Table 1, and the FM stacking always guarantees non-zero $\mathrm{CN}$ for them if each individual material is topologically trivial. It is important to note that $\mathrm{Mn}, \mathrm{Cr}$, Mo, and $\mathrm{W}$ atoms in these compounds that form VdW heterojunctions are all in a $d^{3}$ electronic configuration with $t_{2 g}$ states occupied and $e_{\mathrm{g}}$ states empty. Given the high Curie temperatures (above room temperature) of many compounds in the MXY family (see Table 2), these VdW heterojunctions provide an ideal platform to explore the possibility of room-temperature $\mathrm{QAH}$ state. The electronic structures and topological properties of VdW heterojunctions can be effectively controlled by strain and electric field (see Supplementary Fig. 9). Moreover, the design principles proposed in this work is not limited to this family of materials and can be extended/applied to a large variety of 2D magnetic systems in multiple plane groups protected by various symmetry operations, which will greatly expand the family of heterojunctionbased QAH systems in the future. Therefore, our work will stimulate experimental synthesis and measurement efforts in this class of heterojunction systems and accelerate the discovery and design of new QAH materials, as well as quantum systems for other magnetic topological phases.

\section{Note added}

During the final stage of this work, a related work appeared on arXiv, which performed a systematic search for magnetic topological materials based on magnetic topological quantum chemistry approach ${ }^{52}$.

\section{METHODS}

First-principles calculations based on density functional theory In our calculations, geometric relaxations and total energy calculations were performed using density functional theory within projectoraugmented wave (PAW) potentials $5^{53,54}$ as implemented in the VASP code $^{55}$. A vacuum slab of $20 \AA$ and a plane-wave basis set with an energy cutoff of $520 \mathrm{eV}$ were used. A $10 \times 8 \times 1$ 「-point centered k-point was applied to sample the Brillouin zone. For the initial steps of discovery process, GGA $+U$ was employed to optimize the geometric structures ${ }^{56}$. The $U$-values are $3.25,3.7,3.9,5.3,3.32,6.2,4.38$, and $6.2 \mathrm{eV}$ for $\mathrm{V}, \mathrm{Cr}, \mathrm{Mn}$, $\mathrm{Fe}, \mathrm{Co}, \mathrm{Ni}, \mathrm{Mo}$, and $\mathrm{W}$, respectively, which are outlined in the work by Wang et $a_{1}{ }^{57}$. The structures were fully relaxed until energy and force were converged to $10^{-6} \mathrm{eV}$ and $0.01 \mathrm{eV} / \mathrm{A}$, respectively. The screened hybrid functional of Heyd, Scuseria, and Ernzerhof $(\mathrm{HSEO6})^{58,59}$ was used to compute the band structures of MXY monolayers. For the 10 promising FM MXY scaffolding compounds, HSEO6 calculations were performed to optimize the geometric structures, and compute the magnetic interactions. To verify the dynamical stability of monolayer $\mathrm{MnNF}$ and $\mathrm{MnNCl}$, phonon dispersion analysis was performed by using the density functional perturbation theory method as implemented in the Phonopy code ${ }^{60}$, interfaced with VASP. In phonon and formation energy calculations, GGA $+\mathrm{U}$ along with the PAW potentials were employed, with a convergence criterion of $10^{-8} \mathrm{eV}$ for energy. For the ab initio molecular dynamics (MD) simulations ${ }^{61,62}$, a canonical (NVT) ensemble was used. Time step for MD simulations is $1 \mathrm{fs}$.

\section{DATA AVAILABILITY}

The authors declare that the main data supporting the findings of this study are available within the paper and its Supplementary files. Other relevant data are available from the corresponding author upon reasonable request.

Received: 27 May 2020; Accepted: 4 September 2020; Published online: 13 October 2020 
2. Wang, J., Lian, B. \& Zhang, S.-C. Quantum anomalous Hall effect in magnetic topological insulators. Phys. Scr. T164, 014003 (2015).

3. Chang, C.-Z. \& Li, M. Quantum anomalous Hall effect in time-reversal-symmetry breaking topological insulators. J. Phys. Condens. Matter 28, 123002 (2016).

4. Wu, J., Liu, J. \& Liu, X.-J. Topological spin texture in a quantum anomalous Hall insulator. Phys. Rev. Lett. 113, 136403 (2014).

5. Zhang, R.-X., Hsu, H.-C. \& Liu, C.-X. Electrically tunable spin polarization of chiral edge modes in a quantum anomalous Hall insulator. Phys. Rev. B 93, 235315 (2016).

6. Chen, C.-Z., Xie, Y.-M., Liu, J., Lee, P. A. \& Law, K. T. Quasi-one-dimensional quantum anomalous Hall systems as new platforms for scalable topological quantum computation. Phys. Rev. B 97, 104504 (2018).

7. Zeng, Y., Lei, C., Chaudhary, G. \& MacDonald, A. H. Quantum anomalous Hall Majorana platform. Phys. Rev. B 97, 081102 (2018).

8. $\mathrm{Yu}, \mathrm{R}$. et al. Quantized anomalous Hall effect in magnetic topological insulators. Science 329, 61-64 (2010).

9. Chang, C.-Z. et al. High-precision realization of robust quantum anomalous Hall state in a hard ferromagnetic topological insulator. Nat. Mater. 14, 473-477 (2015).

10. Chang, C.-Z. et al. Experimental observation of the quantum anomalous Hall effect in a magnetic topological insulator. Science 340, 167-170 (2013).

11. Checkelsky, J. et al. Trajectory of the anomalous Hall effect towards the quantized state in a ferromagnetic topological insulator. Nat. Phys. 10, 731 (2014).

12. Bestwick, A. J. et al. Precise quantization of the anomalous Hall effect near zero magnetic field. Phys. Rev. Lett. 114, 187201 (2015).

13. Li, M. et al. Proximity-driven enhanced magnetic order at ferromagneticinsulator-magnetic-topological-insulator interface. Phys. Rev. Lett. 115, 087201 (2015).

14. Tang, $\mathrm{C}$. et al. Above $400-\mathrm{K}$ robust perpendicular ferromagnetic phase in a topological insulator. Sci. Adv. 3, e1700307 (2017).

15. Katmis, F. et al. A high-temperature ferromagnetic topological insulating phase by proximity coupling. Nature 533, 513-516 (2016).

16. Fu, H., Liu, C.-X. \& Yan, B. Exchange bias and quantum anomalous Hall effect in the $\mathrm{MnBi}_{2} \mathrm{Te}_{4}-\mathrm{Crl}_{3}$ heterostructure. Sci. Adv. 6, eaaz0948 (2020).

17. Zou, R., et al. Intrinsic quantum anomalous Hall phase induced by proximity in germanene/ $\mathrm{Cr}_{2} \mathrm{Ge}_{2} \mathrm{Te}_{6}$ van der Waals heterostructure. Phys. Rev. B. 101, 161108 (2020)

18. $\mathrm{Li}$, J. et al. Intrinsic magnetic topological insulators in van der Waals layered $\mathrm{MnBi}_{2} \mathrm{Te}_{4}$-family materials. Sci. Adv. 5, eaaw5685 (2019).

19. Otrokov, M. M. et al. Prediction and observation of an antiferromagnetic topological insulator. Nature 576, 416-422 (2019).

20. Liu, C., et al. Quantum phase transition from axion insulator to Chern insulator in $\mathrm{MnBi}_{2} \mathrm{Te}_{4}$. Preprint at https://ui.adsabs.harvard.edu/abs/2019arXiv190500715L (2019).

21. Deng, Y. et al. Quantum anomalous Hall effect in intrinsic magnetic topological insulator $\mathrm{MnBi}_{2} \mathrm{Te}_{4}$. Science 367, 895-900 (2020).

22. Ge, J., et al. High-chern-number and high-temperature quantum Hall effect without Landau levels. Natl. Sci. Rev. 7, 1280 (2020).

23. Serlin, M. et al. Intrinsic quantized anomalous Hall effect in a moiré heterostructure. Science 367, 900-903 (2020).

24. Sharpe, A. L. et al. Emergent ferromagnetism near three-quarters filling in twisted bilayer graphene. Science 365, 605 (2019).

25. Choudhary, K., Garrity, K. F., Jiang, J., Pachter, R. \& Tavazza, F. Computational search for magnetic and non-magnetic $2 \mathrm{D}$ topological materials using unified spin-orbit spillage screening. NPJ Comput. Mater. 6, 1-8 (2020).

26. Olsen, T. et al. Discovering two-dimensional topological insulators from highthroughput computations. Phys. Rev. Mater. 3, 024005 (2019).

27. Liu, C., Hughes, T. L., Qi, X.-L., Wang, K. \& Zhang, S.-C. Quantum spin Hall effect in inverted Type-II semiconductors. Phys. Rev. Lett. 100, 236601 (2008).

28. Zhang, H., Xu, Y., Wang, J., Chang, K. \& Zhang, S.-C. Quantum spin Hall and quantum anomalous Hall states realized in junction quantum wells. Phys. Rev. Lett. 112, 216803 (2014).

29. Turner, A. M., Zhang, Y., Mong, R. S. K. \& Vishwanath, A. Quantized response and topology of magnetic insulators with inversion symmetry. Phys. Rev. B 85, 165120 (2012).

30. Hughes, T. L., Prodan, E. \& Bernevig, B. A. Inversion-symmetric topological insulators. Phys. Rev. B 83, 245132 (2011).

31. Bradlyn, B. et al. Topological quantum chemistry. Nature 547, 298-305 (2017).

32. Po, H. C., Vishwanath, A. \& Watanabe, H. Symmetry-based indicators of band topology in the 230 space groups. Nat. Commun. 8, 50 (2017).

33. Kruthoff, J., de Boer, J., van Wezel, J., Kane, C. L. \& Slager, R.-J. Topological classification of crystalline insulators through band structure combinatorics. Phys. Rev. X 7, 041069 (2017)

34. Bergerhoff, G., Hundt, R., Sievers, R. \& Brown, I. The inorganic crystal structure data base. J. Chem. Inf. Comput. Sci. 23, 66-69 (1983).
35. Jain, A. et al. Commentary: The Materials Project: a materials genome approach to accelerating materials innovation. APL Mater. 1, 011002 (2013).

36. Gražulis, S. et al. Crystallography Open Database (COD): an open-access collection of crystal structures and platform for world-wide collaboration. Nucleic Acids Res. 40, D420-D427 (2011).

37. Ashton, M., Paul, J., Sinnott, S. B. \& Hennig, R. G. Topology-scaling identification of layered solids and stable exfoliated 2D materials. Phys. Rev. Lett. 118, 106101 (2017).

38. Choudhary, K., Kalish, I., Beams, R. \& Tavazza, F. High-throughput identification and characterization of two-dimensional materials using density functional theory. Sci. Rep. 7, 5179 (2017).

39. Haastrup, S. et al. The computational 2D materials database: high-throughput modeling and discovery of atomically thin crystals. 2D Mater. 5, 042002 (2018).

40. Mounet, N. et al. Two-dimensional materials from high-throughput computational exfoliation of experimentally known compounds. Nat. Nanotechnol. 13, 246 (2018).

41. Zhou, J. et al. 2DMatPedia, an open computational database of two-dimensional materials from top-down and bottom-up approaches. Sci. Data 6, 86 (2019).

42. Zhang, T. et al. Catalogue of topological electronic materials. Nature 566, 475-479 (2019).

43. Tang, F., Po, H. C., Vishwanath, A. \& Wan, X. Comprehensive search for topological materials using symmetry indicators. Nature 566, 486-489 (2019).

44. Vergniory, M. et al. A complete catalogue of high-quality topological materials. Nature 566, 480 (2019).

45. Guo, Y., Zhang, Y., Yuan, S., Wang, B. \& Wang, J. Chromium sulfide halide monolayers: intrinsic ferromagnetic semiconductors with large spin polarization and high carrier mobility. Nanoscale 10, 18036-18042 (2018).

46. Qi, J., Wang, H. \& Qian, X. Electrically tunable, high Curie temperature 2D ferromagnetism in van der Waals layered crystals. Appl. Phys. Lett. 117, 083102 (2020).

47. Wang, C. et al. A family of high-temperature ferromagnetic monolayers with locked spin-dichroism-mobility anisotropy: $\mathrm{MnNX}$ and $\mathrm{CrCX}(\mathrm{X}=\mathrm{Cl}, \mathrm{Br}, \mathrm{I} ; \mathrm{C}=\mathrm{S}$, Se, Te). Sci. Bull. 64, 293-300 (2019).

48. Jiang, Z., Wang, P., Xing, J., Jiang, X. \& Zhao, J. Screening and design of novel 2D ferromagnetic materials with high Curie temperature above room temperature. ACS Appl. Mater. Inter. 10, 39032-39039 (2018).

49. Xiang, H., Lee, C., Koo, H.-J., Gong, X. \& Whangbo, M.-H. Magnetic properties and energy-mapping analysis. Dalton Trans. 42, 823-853 (2013)

50. Xiang, H., Kan, E., Wei, S.-H., Whangbo, M.-H. \& Gong, X. Predicting the spin-lattice order of frustrated systems from first principles. Phys. Rev. B 84, 224429 (2011).

51. Grimme, S., Antony, J., Ehrlich, S. \& Krieg, H. A consistent and accurate ab initio parametrization of density functional dispersion correction (DFT-D) for the 94 elements H-Pu. J. Chem. Phys. 132, 154104 (2010).

52. $\mathrm{Xu}, \mathrm{Y}$., et al. High-throughput Calculations of antiferromagnetic topological materials from magnetic topological quantum chemistry. Preprint at https://ui. adsabs.harvard.edu/abs/2020arXiv200300012X (2020)

53. Blöchl, P. E. Projector augmented-wave method. Phys. Rev. B 50, 17953 (1994).

54. Kresse, G. \& Joubert, D. From ultrasoft pseudopotentials to the projector augmented-wave method. Phys. Rev. B 59, 1758 (1999).

55. Kresse, G. \& Furthmüller, J. Efficiency of ab-initio total energy calculations for metals and semiconductors using a plane-wave basis set. Comput. Mater. Sci. 6 15-50 (1996).

56. Dudarev, S. L., Botton, G. A., Savrasov, S. Y., Humphreys, C. J. \& Sutton, A. P. Electron-energy-loss spectra and the structural stability of nickel oxide: An LSDA +U study. Phys. Rev. B 57, 1505-1509 (1998)

57. Wang, L., Maxisch, T. \& Ceder, G. Oxidation energies of transition metal oxides within the GGA+U framework. Phys. Rev. B 73, 195107 (2006).

58. Heyd, J., Scuseria, G. E. \& Ernzerhof, M. Hybrid functionals based on a screened Coulomb potential. J. Chem. Phys. 124, 219906 (2006).

59. Heyd, J., Scuseria, G. E. \& Ernzerhof, M. Hybrid functionals based on a screened Coulomb potential. J. Chem. Phys. 118, 8207-8215 (2003).

60. Togo, A. \& Tanaka, I. First principles phonon calculations in materials science. Scr. Mater. 108, 1-5 (2015)

61. Kresse, G. \& Hafner, J. Ab initio molecular dynamics for liquid metals. Phys. Rev. $B$ 47, 558-561 (1993).

62. Kresse, G. \& Hafner, J. Ab initio molecular-dynamics simulation of the liquidmetal-amorphous-semiconductor transition in germanium. Phys. Rev. B 49, 14251-14269 (1994).

\section{ACKNOWLEDGEMENTS}

We thank Cui-zu Chang and Xiaodong Xu for the helpful discussion. J.P. and Q.Y. acknowledge support from the U.S. Department of Energy under Award \#DESC0019275 for the design of data-driven discovery pipeline and the first-principles computational work. J.Y. and C.X.L. acknowledge the support of DOE grant (DESC0019064) for the analytical model and symmetry analysis, and the Office of Naval 
Research (Grant number N00014-18-1-2793), as well as Kaufman New Initiative research grant of the Pittsburgh Foundation. A.J. acknowledges support from U.S. DOE SE-SC0014388. S.X.D. thanks the International Partnership Program of Chinese Academy of Sciences, Grant number 112111KYSB20160061. It benefitted from the supercomputing resources of the National Energy Research Scientific Computing Center (NERSC), a U.S. Department of Energy Office of Science User Facility operated under Contract number DE-AC02-05CH11231.

\section{AUTHOR CONTRIBUTIONS}

Q.Y. and C.X.L. conceived and coordinated the research project. J.B.P. and J.Y. contributed equally to this work. J.B.P., Y.F.Z., and Q.Y. implemented the discovery workflow and performed the DFT calculations. J.Y. and C.X.L. performed the symmetry analysis and constructed the effective and tight-binding models. All authors participated in discussing the data and editing the manuscript.

\section{COMPETING INTERESTS}

The authors declare no competing interests.

\section{ADDITIONAL INFORMATION}

Supplementary information is available for this paper at https://doi.org/10.1038/ s41524-020-00419-y.
Correspondence and requests for materials should be addressed to C.-X.L. or Q.Y.

Reprints and permission information is available at http://www.nature.com/ reprints

Publisher's note Springer Nature remains neutral with regard to jurisdictional claims in published maps and institutional affiliations. Attribution 4.0 International License, which permits use, sharing, adaptation, distribution and reproduction in any medium or format, as long as you give appropriate credit to the original author(s) and the source, provide a link to the Creative Commons license, and indicate if changes were made. The images or other third party material in this article are included in the article's Creative Commons license, unless indicated otherwise in a credit line to the material. If material is not included in the article's Creative Commons license and your intended use is not permitted by statutory regulation or exceeds the permitted use, you will need to obtain permission directly from the copyright holder. To view a copy of this license, visit http://creativecommons. org/licenses/by/4.0/.

(c) The Author(s) 2020 